Davis GK, O'Sullivan A, Mangos G, Homer CSE, Craig M, Harvey SB, Henry A, Pettit F, Roberts L, Brown MA. (2016) Postpartum Physiology, Psychology and Paediatric Follow Up Study (P4 Study) - Study Protocol. Pregnancy Hypertension: An International Journal of Women's Cardiovascular Health. doi.org/10.1016/j.preghy.2016.08.241. Available from:

http://www.sciencedirect.com/science/article/pii/S2210778916300319

\title{
Postpartum Physiology, Psychology and Paediatric Follow Up Study (P4 Study) - Study Protocol
}

A/Prof Gregory K Davis ${ }^{1,2}$ Greg.Davis@health.nsw.gov.au (Corresponding Author) Ms Lynne Roberts ${ }^{1,3}$ Lynne.Roberts2@health.nsw.gov.au

A/Prof George Mangos ${ }^{1,2}$ g.mangos@unsw.edu.au

Dr Amanda Henry ${ }^{1,2}$ Amanda.Henry@unsw.edu.au

Dr Franziska Pettit ${ }^{1,2}$ Franziska.Pettit@health.nsw.gov.au

Prof Anthony O'Sullivan ${ }^{1,2}$ a.osullivan@unsw.edu.au

Prof Caroline SE Homer ${ }^{1,3}$ Caroline.Homer@uts.edu.au

Prof Maria Craig ${ }^{1,2,4}$ m.craig@unsw.edu.au

A/Prof Samuel B Harvey ${ }^{1,2,5}$ s.harvey@unsw.edu.au

Prof Mark A Brown ${ }^{1,2}$ m.brown@unsw.edu.au

1. St George Hospital, Gray Street, Kogarah, NSW 2217. Australia

2. University of NSW, Randwick NSW 2031, Australia

3. University of Technology Sydney, Broadway, Ultimo NSW 2007. Australia

4. Sydney Children's Hospital, Randwick, NSW 2031. Australia

5. Black Dog Institute, Randwick, NSW 2031. Australia

\section{Funding}

Funding has been provided by St George and Sutherland Medical Research Foundation.

\section{List of abbreviations}

ABPM - 24 hour Ambulatory Blood Pressure Monitoring; ASQ - Ages and Stages

Questionnaire; BP - Blood Pressure; CVD - Cardiovascular Disease; EPDS - Edinburgh

Postnatal Depression Scale; GAD-7 - General Anxiety Disorder 7-item scale; MIB - Mother Infant Bonding; P4 Study - Postpartum Physiology, Psychology and Paediatric Follow up Study; PDS - Posttraumatic Diagnostic Scale; SD - Standard Deviation 


\begin{abstract}
:
Background

Women who have had hypertension in pregnancy are at greater risk of long term cardiovascular disease (CVD). Little is known about their cardiovascular risk postpartum or the effects on the woman's mental health and the outcomes of their infants. In this project we will study the physiological and psychological health of women and the physical health and development of their infants six months, two years and five years after birth. We will establish normal blood pressure (BP) ranges for women who were normotensive in pregnancy and use these to assess BP in women who had gestational hypertension (GH) or preeclampsia (PE).
\end{abstract}

Design/Methods

Women will be asked to participate if they have given birth in the preceding six months. They will be excluded if they had diabetes, hypertension, renal or other serious maternal disease prior to pregnancy or congenital anomaly in the pregnancy. We will recruit 292 women who were normotensive and their babies, 100 who had PE and 100 who had GH and their babies. They will be assessed at six months, two and five years after birth. At each assessment mothers will have their blood pressure (BP) assessed peripherally with a liquid crystal sphygmomanometer and 24 hour ambulatory blood pressure monitoring (ABPM), and centrally with non-invasive applanation tonometry. Additional physiological testing will include: body composition; energy balance; vascular compliance; cardiac function; liver and renal function, lipids and biochemistry; glucose and insulin; and urinalysis. Psychological status will be assessed with validated self-report questionnaires for depression, anxiety, posttraumatic stress disorder and mother-infant bonding. The babies will have a medical examination by a paediatrician at each assessment. Their behavioural development will be assessed with an Ages and Stages Questionnaire completed by their mother at each assessment and a developmental assessment by a child psychologist at two and five years.

\title{
Conclusions:
}

This study will re-define normal BP and other physiological parameters for young parous women thereby permitting a more sensitive assessment of post-partum BP and other cardiovascular risk markers in women who have had GH or PE. It will also determine the extent, if any, of psychological disorders in these women and developmental or other concerns in their babies.

Trials Registration:

Australian and New Zealand Clinical Trials Registry Number: ACTRN12613001260718

Keywords

Preeclampsia, cardiovascular risk, postpartum risk, postnatal depression, infant outcomes 
Highlights

- Establishes a protocol for a prospective postpartum follow up study

- Includes women who were normotensive and hypertensive in pregnancy and their babies

- Incorporates Physiological and psychological assessment of mothers after defining new 'normal ranges' for women who have had normal pregnancies

- Includes Physical and developmental assessment of their babies

- Follow up at six months, two and five years postpartum

\section{Introduction:}

Hypertensive diseases in pregnancy are the most common medical complication of pregnancy affecting $10 \%$ of pregnant women [1]. GH is usually a benign condition with good maternal and fetal outcomes but $4 \%$ of pregnant women will develop PE, with increased maternal and fetal morbidity and mortality [2]. Cardiovascular disease is the leading cause of death for women in the western world [3]. Women who have had hypertension in pregnancy have a 2-3 fold increased risk of CVD in later life [4] and an increased risk for venous thromboembolism, diabetes, microalbuminuria, end stage renal disease [5] and mental health disorders [6]. The significant physiological demands of pregnancy are a stress test [7] that either unmasks a predisposition to CVD [8,9] or initiates a cascade of vascular changes which lead to later CVD [10].

\section{Hypertension and Metabolic Syndrome after Hypertension in Pregnancy}

Subgroups of women with hypertension in pregnancy and CVD share the features of the metabolic syndrome including obesity, hypertension, insulin resistance and dyslipidaemia, and preeclampsia has been described as the metabolic syndrome of pregnancy [10]. This common risk pattern is relevant to clinical practice in that, although women with early onset PE (prior to 34 weeks gestation) have a greater risk of subsequent CVD [11] and end stage kidney disease [12], the features of the metabolic syndrome are more common in women with late onset PE (after 34 weeks gestation) or GH $[13,14]$.

Thus, women with any form of PE or even GH should be considered at risk for future CVD. The diagnosis of hypertension in pregnancy therefore provides a unique opportunity to identify a group of young women at risk of later disease and to institute measures to potentially lower their long term risk $[15,16]$. Interventional studies examining exercise and dietary modification in the postpartum period have been shown to be effective, particularly in women who know they are at increased risk $[17,18]$. However data are urgently needed to determine prevention strategies, screening and treatment schedules for the large number of women who have had hypertension in pregnancy [16]. Perhaps the biggest barrier to date is our need to rely on cardiovascular risk assessments that are designed for 
use in the general population, usually older people and often male predominant $[19,20]$. For example, we have shown previously that women who have had hypertension in pregnancy have higher BP several years post-partum than women who had a normotensive pregnancy [21]. Nonetheless their BP levels fall within the so-called 'normal' range and are therefore unlikely to be recognised as having a BP higher than that expected for a young woman who has had a previously normal pregnancy.

What is needed is a study to define normal parameters for blood pressure and metabolic function in: 1) normal postpartum women; and 2) women who had hypertension in pregnancy; to determine where women who have had hypertension in pregnancy fall in newly defined 'normal' ranges. This is a major knowledge gap: there are no published data to provide guidance to clinicians. To the best of our knowledge this has not been done to date. Definition of the group of women at risk, i.e. falling outside newly defined normal parameters for this age group of women, would allow the establishment of a follow up guidance to coordinate early intervention for modification of lifestyle factors and treatment of hypertension and insulin resistance/diabetes [22,23].

\section{Psychological Issues after Hypertension in Pregnancy}

Our society generally assumes that the birth of a child should be an entirely positive experience. While on balance this is usually true, these expectations mean that women may find it difficult to discuss some of their negative experiences. This is important as childbirth can be difficult, with $20-48 \%$ of women reporting their birth experience as traumatic [24]. At one extreme, pregnancy complications and a difficult childbirth can lead to PTSD [25], although the exact prevalence of PTSD among new mothers is uncertain [26]. Not surprisingly, PTSD appears to be more common where there have been complications in pregnancy and/or the birth, such as PE [27] and co-existing maternal depression further increases the risk of PTSD [28].

PTSD is one of a number of mental disorders that can be seen following a complicated pregnancy and birth. Increased levels of anxiety and depression have been reported amongst postpartum women, although it is not clear whether these symptoms develop during or after the pregnancy [5].

Gaining a better understanding of the psychological consequences of complicated pregnancies is crucial for a number of reasons. Firstly, apart from the impact of these psychological disorders on the mother, maternal PTSD, anxiety and depression can affect the emotional and behavioural development of the infant and disturb mother-infant attachment [29]. Secondly, there is increasing evidence that if groups at high risk of mental disorder can be identified, targeted interventions can prevent around $25 \%$ of the cases of 
mental disorder occurring [30]. However, the success of such preventative interventions relies on a valid way of predicting those at increased risk.

\section{Paediatric Outcomes after Hypertension in Pregnancy}

While it is accepted that PE increases perinatal morbidity and mortality [2], the effects on the subsequent health and development of the infants of women who have had hypertension in pregnancy have not been adequately addressed. Variable findings have been reported [31,32]. Minor cognitive differences at two years of age were noted in infants of mothers with hypertension in pregnancy born at less than 35 weeks gestation [33] and a significant delay was reported in the children of women who had PE in a study of neurodevelopmental disability at two years of age [34]. In a study of developmental outcomes at two years of age in babies born at less than 32 weeks gestation, those infants whose mothers had PE were more likely to have mild cognitive impairment (OR $10.9,95 \% \mathrm{Cl}$ $1.4,84.9)$ [35]. Similarly, in a small study of growth restricted babies (<5th centile) at three years of age, infants of mothers who had PE were more likely to have a moderately impaired cognitive development (mean IQ 85.5 vs. 96.9, p<0.03) [36]. However in a long term epidemiological study of recruits into the Israeli armed forces at 17 years of age there was no difference in mean intelligence scores between those whose mothers had PE and those who did not [37]. The study design is a longitudinal follow up of infants born to mothers with and without hypertension, which will provide sufficiently powered, controlled data to assess the effect of hypertension during pregnancy on growth and development, with adjustment for risk modifying variables, particularly gestational age.

With this study we will define for the first time what should be considered normal BP ( $A B P M$, routine office and central BP), lipids, heart and kidney function and mental health scores in young women who have had a normal pregnancy and the health of their babies. Similarly we will determine which women who were hypertensive in pregnancy and/or their babies who are at increased later risk.

\section{Methods/Design}

\section{Study Design and Setting}

A prospective, open, cohort study is being undertaken at St George Hospital, a large metropolitan teaching hospital that serves an area of Sydney, Australia, with a diverse ethnic community.

\section{Hypotheses}

1. That women who have had hypertension in pregnancy:

a. Have risk factors for CVD that are only recognised by comparison with a new 'normal' range for blood pressure and other cardiovascular risk factors.

b. Have a higher prevalence of psychological morbidity postpartum 
2. That the offspring of women who have had hypertension in pregnancy have a greater likelihood of abnormal growth and development six months after birth

\section{Outcomes}

1. The primary outcome is the prevalence of women in the formerly hypertensive groups with mean 24 hour systolic or diastolic BP two standard deviations greater than the mean BP derived from women who had normal pregnancies.

2. Secondary outcomes are: (1) physiological measures: central BP, body composition, energy balance, cardiac function, renal function, lipids, insulin resistance (HOMA score) and urinalysis; (2) psychological measures: depression, anxiety, post-traumatic stress and mother-infant bonding; and (3) infant health: physical health and development.

3. Development of guidance for the assessment, treatment and follow up of:

a. Women who have increased risk factors for later CVD.

b. Women who have psychological morbidity after hypertension in pregnancy.

c. Children with physical or developmental impairment after maternal hypertension in pregnancy.

\section{Ethics Approval}

The study has been approved by the South Eastern Sydney Local Health District Human Research Ethics Committee (reference number: 12/195(HREC/12/POWH/395)

\section{Entry Criteria}

Women will be eligible if they have given birth to a live baby within six months and have a good understanding of written and spoken English. The babies born to these women will be included in the study. Women will be excluded if they had diabetes, hypertension, renal or other serious maternal disease prior to pregnancy, they had a multiple pregnancy, their baby was born with a congenital anomaly or they are pregnant again.

\section{Study Entry and Consent}

1. Women who are inpatients on the postnatal ward will be spoken to by the Research Midwife and given the Participant Information and Consent Form to read. If a woman agrees to participate in the study, written informed consent for the woman and her child will be obtained and an appointment made for the Six Month Assessment.

2. Women who are discharged prior to meeting the Research Midwife will be mailed an Invitation to be Part of the Study letter and the Participant Information and Consent Form and a follow up phone call made. Written consent for the woman and her child will be obtained when she attends the Six Month Assessment. 


\section{Measurements:}

\section{Physiological measures - women}

1. Peripheral $B P$ will be measured using a hybrid liquid crystal sphygmomanometer (mean of three readings) and ABPM, as the latter is known to more reliably predict long term CVD risk [38] and our previous work has shown subtle differences which would not have been detected with routine intermittent auscultation [21].

2. Body composition will be determined by bio-impedance analysis [39], using a multifrequency body composition monitor (Fresenius Medical Care, Australia). This technique enables total body water volume, intra- and extracellular water, lean body mass and fat mass to be measured. These measures are relevant to hypertension and the metabolic syndrome.

3. Energy balance will be determined by measuring total energy expenditure over 24 hours using the SenseWear Armband (Temple Healthcare, Australia) which is worn for 24 hours, and measures skin temperature and heat flux. Participants will be asked to complete a three day food diary and the data will be entered into a Nutrient Calculation Program (Foodworks Version 1, Xyris Software Aust. Pty Ltd. Australia) enabling macronutrient dietary intake to be quantified. These measures will provide more detailed understanding of any differences between groups in body weight.

4. Vascular wall stiffness and central BP will be determined using pulse wave analysis assessed with an applanation tonometer (Sphygmocor, AtCor Medical Pty Limited, Australia). Pulse wave analysis is performed over the radial artery, allowing noninvasive measurement of central BP, detection of aortic stiffness and endothelial dysfunction. Endothelial dysfunction is an important component of the pathophysiology of PE and previous studies of pulse wave analysis in pregnancy have shown abnormalities in preeclamptic women $[40,41]$. However there are conflicting results on whether these persist into the postpartum period [42]. Measurement of vascular compliance is of increasing importance in the general hypertensive population and may become part of routine clinical practice in the near future [43]; hence it is important that we define normal for these parameters in this population.

5. Cardiac function will be assessed with transthoracic 2-D echocardiography. Left ventricular (LV) systolic and diastolic function, LV wall thickness and fractional shortening will be measured as markers of cardiac dysfunction. Cardiac function is impaired in some women who have had a pregnancy complicated by hypertension, with persisting abnormal LV geometry and decreased diastolic function [44]. A single cardiologist will report all echocardiograms performed on women in the Study.

6. Blood samples will be taken for full blood count, urea, electrolytes, creatinine and uric acid, liver function tests, magnesium, calcium, phosphate, fasting insulin and glucose, lipids (cholesterol, low and high density lipoproteins and triglycerides), and Vitamin D. An additional $20 \mathrm{~mL}$ of blood will be separated and the serum and plasma stored at $-80^{\circ} \mathrm{C}$ for later assay of sex hormone binding globulin, haemoglobin $\mathrm{A} 1 \mathrm{C}$, 
parathyroid hormone, adiponectin, leptin and angiotensin II receptor autoantibodies (implicated in the pathogenesis of PE [45]).

7. A urine sample will be collected for automated dipstick urinalysis and laboratory testing for albumin, protein and creatinine. Blood and urine testing of these parameters will determine whether subtle abnormalities of renal function, vitamin $D$ or usual cardiovascular risk factors are present in women who have had hypertensive pregnancies.

\section{Psychology}

The assessment of women's psychological health will be made using validated self-report questionnaires. Participants will be asked to complete the Edinburgh Postnatal Depression Scale (EPDS) [46], the General Anxiety Disorder 7-item scale (GAD-7) [47] and the Posttraumatic Diagnostic Scale (PDS) [48]. The EPDS is a 10 item measure designed to screen women for symptoms of emotional distress during pregnancy and the postnatal period which has been validated in Australian women [49]. The GAD-7 is a well validated tool for detecting Generalised Anxiety Disorder, the most common, non-phobic anxiety disorder in a post-natal population, while the PDS is a 49 item scale that enquires about the key diagnostic criteria of post-traumatic stress disorder. In addition, maternal infant bonding will be assessed using the self-report Maternal Infant Bonding (MIB) questionnaire [50].

\section{Infant Assessment}

The infant's development will be assessed using the Ages and Stages Questionnaire (ASQ) that is within one month of their child's age, corrected for the infant's gestation at birth [52]. This is a short questionnaire regarding the child's development that the mother or care-giver completes. A full history and physical examination performed by a specialist Paediatrician will determine the presence of any chronic illness, and elicit information on hospital admissions. Height, weight and head circumference will be plotted on World Health Organisation charts and standard deviation scores determined. Blood pressure will be measured using an appropriately sized paediatric cuff on an automated sphygmomanometer.

\section{Assessment Schedule}

1. Six Months: initial assessment of mothers and babies will occur at six months postpartum. This time was chosen as the maternal physiology and psychology will have returned to normal after pregnancy, babies will usually have recovered from any illness related to the birth and mothers are more likely to be compliant in attending for assessment.

2. Two Years: mothers and babies will be assessed at two years to assess medium term outcomes and that the risk assessment at six months postpartum has been accurate. 
By two years of age subtle physical and developmental impairment in the infants will be more apparent.

3. Five Years: the development of CVD is a long term health risk. Although it is unlikely that there will be overt evidence of CVD at five years, this was deemed to be a realistic long term assessment of the outcomes of mothers and infants.

\section{Six Month Assessment}

Women recruited to the study will be asked to attend six months after birth. Prior to this they are sent, and asked to complete, an ASQ corrected for prematurity. Written consent will be obtained at this visit for them and their baby to take part in the study if not already completed.

Data Collection

1. History

Demographics: age, ethnicity, relationship status, highest educational level, occupations of mother and father

Medical: history of infertility and fertility treatment, psychiatric history, significant illness, diabetes, smoking, renal disease

Family: history of hypertension, CVD, diabetes

Obstetric: diagnosis of GH or $\mathrm{PE}$, gestation at diagnosis, antihypertensive medication score, labour onset, gestation at birth, pain relief in labour, mode of birth, MgSO4 given, episodes of severe hypertension, postpartum complications

2. Measurements

Weight, height, and ratio of waist:hip circumference

Blood pressure - average of three measurements using a hybrid sphygmomanometer Vascular wall stiffness using Sphygmocor applanation tonometer

Body composition using body composition monitor

Blood and urine testing

3. Psychological testing

Self-report questionnaires - EPDS, PDS, GAD-7, MIB scales

\section{Follow up appointments}

Follow up will be arranged for the collection of the food diary and ABPM and appointments for the SenseWear Armband and food diary, echocardiogram and the visit to the Paediatrician.

\section{Cardiac Function}

Echocardiography will be performed at a separate appointment. The parameters measured will be: LV geometry including wall thickness, regional wall thickness and LV mass index, diastolic parameters, and global longitudinal strain. All reporting of echocardiograms on participants in the P4 Study will be completed by one cardiologist. 


\section{Two and Five Year Assessments}

All mothers seen for Six Month Assessments will be contacted at 6, 12 and 18 months by a member of the P4 Study team to confirm their current residential address and document any changes of contact details for themselves and the contact people they provide at trial entry. Appointments will be made for participants at two and five years postpartum. An age appropriate ASQ will be posted for the mother to complete prior to the visit.

All physiological and psychological testing will be repeated as in the Six Month Assessment visit. An appointment will be made for children in the study to be examined by the paediatrician and a Child Developmental Psychologist at two and five years. The psychologist will carry out a Bayley III assessment [52] at two years and full developmental assessment at five years to determine the child's development in the areas of cognitive, language and communication, and both fine and gross motor skills.

\section{Management of abnormal findings}

With consent, a summary of the study results will be sent to the woman's family doctor highlighting abnormal findings. If abnormalities are detected the woman will be contacted and advised to visit her doctor. Referral to Mental Health Services will be offered for abnormal score/s on any of the mental health questionnaires.

\section{Sample Size}

The study will be powered on the proportion of average 24 hour average diastolic blood pressure (DBP) readings two or more standard deviations (SD) above the mean for women who were normotensive in pregnancy. In preliminary data on normotensive women at six months postpartum, 4/98 (4\%) had 24 hour average DBP readings $>2$ SD above the mean, as expected, compared to 5/22 (23\%) hypertensive women. Based on these data, 56 women will be required in each group, with a $95 \%$ confidence level and $85 \%$ power. Sample size assessment is based on the hypertensive group as a whole but current data suggests that the women who had PE and GH have similar rates of elevated average 24 hour DBP. We plan to recruit 292 women who were normotensive in pregnancy in order to construct a 95\% reference range [53] for normal values in a postpartum population. One hundred women who had GH and 100 who had PE in pregnancy will be recruited. This will allow for an estimated $15 \%$ loss to follow up and women who will be pregnant again prior to two year and five year follow ups. Women who have been pregnant again prior to follow up at two and five years will be analysed on intention-to-treat with subgroup analysis.

\section{Data Analysis}

Initial analyses of the two groups will be descriptive, and the appropriate Chi-squared test used for categorical data and Student's t-test for continuous data when normally 
distributed. More sophisticated analyses will include, where relevant, the use of (i) generalised linear models to evaluate the effects of covariates and their interactions, and (ii) the method of generalised estimating equations to accommodate the repeated measures as well as covariates for each woman. A probability of $<0.05$ will be considered statistically significant.

\section{Discussion}

ABPM is currently the gold standard for BP measurement and a key determinant of cardiovascular risk [38]. The primary outcome of the study is the mean 24 hour diastolic BP at six months postpartum as a surrogate marker for long term risk of CVD. We will recruit 292 women who were normotensive in pregnancy to define normal values and ranges for all parameters. We will assess of 100 women who had GH and 100 with PE in their pregnancy to determine the frequency of abnormal findings in these women and their babies. Of great importance, it will also allow us to reassure those women who are not at long term risk of CVD.

This study is of significant clinical and public health importance because of the recently recognised long term cardiovascular and mental health consequences of women who have had hypertensive disease in pregnancy. There are no comparable follow up studies of women and their babies underway, nationally or internationally. In particular, clinicians presently do not have an upper limit of normal for cardiovascular risk or psychological parameters in young women who have had a normal pregnancy; we rely upon 'normal' values for parameters such as BP that have been derived from the general, often older, population. At the end of this study we will define for the first time in young women who have had a normal pregnancy what should be considered normal BP (by ABPM, routine office and central BP measures), blood lipids, heart and kidney function and mental health scores and the health of their babies. Our ultimate goal is to establish follow up guidance for women who have had hypertension in pregnancy and their babies, with the guidance underpinned by the findings of this research project [22].

\section{Acknowledgements}

Dr James Scandol for statistical advice

Dr Joseph Khouri for paediatric medical assessments

Dr Clare Rowe for paediatric developmental assessments 